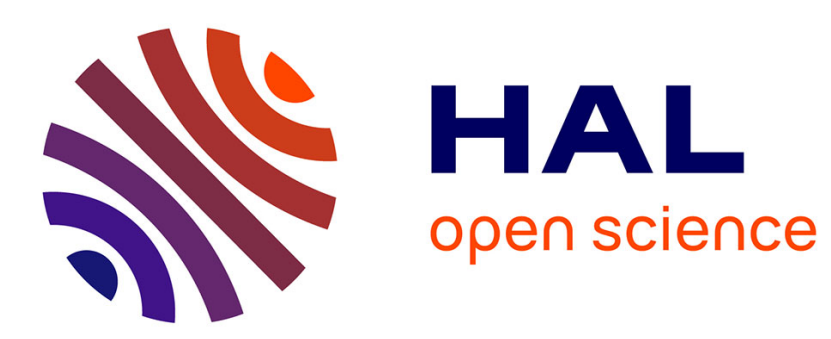

\title{
High-pressure structural changes in liquid silica
}

A. Denoeud, S. Mazevet, F. Guyot, F. Dorchies, J. Gaudin, A. Ravasio, E.

Brambrink, A. Benuzzi-Mounaix

\section{To cite this version:}

A. Denoeud, S. Mazevet, F. Guyot, F. Dorchies, J. Gaudin, et al.. High-pressure structural changes in liquid silica. Physical Review E , 2016, 94 (3), pp.031201(R). 10.1103/PhysRevE.94.031201 . hal01393738

\section{HAL Id: hal-01393738 \\ https: / hal.sorbonne-universite.fr/hal-01393738}

Submitted on 8 Nov 2016

HAL is a multi-disciplinary open access archive for the deposit and dissemination of scientific research documents, whether they are published or not. The documents may come from teaching and research institutions in France or abroad, or from public or private research centers.
L'archive ouverte pluridisciplinaire HAL, est destinée au dépôt et à la diffusion de documents scientifiques de niveau recherche, publiés ou non, émanant des établissements d'enseignement et de recherche français ou étrangers, des laboratoires publics ou privés. 


\title{
High Pressure Structural Changes in Liquid Silica
}

\author{
A. Denoeud ${ }^{1,2}$, S. Mazevet ${ }^{3,4}$, F. Guyot ${ }^{5}$, F. Dorchies ${ }^{6}$, J. Gaudin $^{6}$, A. Ravasio ${ }^{1,2}$, \\ E. Brambrink ${ }^{1,2}$, and A. Benuzzi-Mounaix ${ }^{1,2}$ \\ ${ }^{1}$ LULI - CNRS, Ecole Polytechnique, CEA, Université Paris-Saclay, F-91128 Palaiseau cedex, France \\ ${ }^{2}$ Sorbonne Universités, UPMC Univ Paris 06, CNRS, laboratoire d'utilisation des lasers intenses (LULI), place Jussieu, 75252 Paris cedex 05, France \\ ${ }^{3}$ LUTH, Observatoire de Paris, CNRS, Université Paris Diderot, 92195 Meudon, France \\ ${ }^{4}$ Département de Physique Théorique et Appliquée, CEA, 91680 Bruyère-le-Chatel, France \\ ${ }^{5}$ Institut de Minéralogie de Physique des Matériaux (IMPMC), Sorbonne Universités MNHN. UPMC, IRD, Paris, France \\ ${ }^{6}$ Centre Lasers Intenses et Applications (CELIA), CNRS, CEA, Université Bordeaux 1, 33405 Talence, France
}

\begin{abstract}
The structural properties of liquid silica at high pressure and moderate temperature conditions, also refereed to as the Warm Dense Matter (WDM) regime, were investigated using time resolved K edge X-ray absorption spectroscopy and ab initio calculations. We used a nanosecond laser beam to compress uniformly a solid $\mathrm{SiO}_{2}$ target and a picosecond laser beam to generate a broad band $\mathrm{X}$-ray source. We obtained X-ray absorption spectra at the Si K-edge over a large pressure-temperature domain to probe the liquid phase up to 3.6 time normal solid density. Using $a b$ initio simulations, we are able to interpret the changes of the X-ray Absorption Near Edge Structure (XANES) with increasing densities as an increase of the coordination number of silicon by oxygen atoms from four to nine. This indicates that up to significant temperatures, the liquid structure becomes akin to what is found in the solid $\mathrm{SiO}_{2}$ phases.
\end{abstract}

The continuous discovery of very diverse exoplanets turns the modeling of their interiors as a new scientific challenge [1]. In this context, it is now critical to expand our knowledge of the equations of state and of the physical properties of key constituents such as hydrogen, helium, water, silicates, and iron at multi-Mbar pressures and few-eV temperatures [2-5]. Among these constituents, complex silicates (i.e. $(\mathrm{Mg}, \mathrm{Fe}) \mathrm{SiO}_{3}$ and $\left.(\mathrm{Mg}, \mathrm{Fe})_{2} \mathrm{SiO}_{4}\right)$ and the products of their dissociation $\left(\mathrm{SiO}_{2}, \mathrm{MgO}, \mathrm{FeO}\right)$ are of major interest for terrestrial exoplanets that can reach up to 10 earth mass. Nevertheless, little is known on the properties of silicates at pressures greater than those encountered in the Earth mantle [6]. Their metallization, dissociation, and structural properties constitute a central issue for the modeling of terrestrial planets or the cores of giant and icy giant planets [7-10]. In particular, silica is an insulator with a large electronic band gap at normal density-temperature conditions [11] and constitutes an important end-member of complex silicates, since it is likely produced by the dissociation of highly-compressed $\mathrm{MgSiO}_{3}$ [7]. Consequently, its study in the warm dense matter regime constitutes a first step for a validation of the silicates phase diagram relevant for planetary models.

The metallization and melting of $\mathrm{SiO}_{2}$ have been investigated along the principal shock Hugoniot by using laser shock experiments [12-13-14]. For metallization, reflectivity data were found in good agreement with $a b$ initio calculations [15]. These studies suggested a close connection between the increase in reflectivity, the closing of the band gap and the loss of correlation between the Si and $\mathrm{O}$ atoms. They led to a simple phase diagram of liquid silica with two regimes [13]: a so called bonded liquid characterized by a mixture of various structural units based on $\mathrm{Si}-\mathrm{O}$ bonds, and an highly electrically conducting atomic fluid (Fig. 1).

These hypotheses were based on indirect measurements, namely reflectivity, temperature and shock velocity. The structural information and the resulting phase diagram were deduced from macroscopic measurements [13]. Following our recent work on the metallization of fused silica at Mbar pressures [16], we go a step further here by using a structural diagnostic, based on X-ray absorption spectroscopy. Our aim was to directly access the evolution of the electronic and ionic structures of liquid silica at high densities and temperature. To this end, we obtained experimental and theoretical X-ray Absorption Near Edge Structure (XANES) data of highly compressed liquid silica (up to $\rho / \rho_{0} \approx 3.6$, corresponding to pressures $\approx 14 \mathrm{Mbar}$ ). We show that liquid $\mathrm{SiO}_{2}$ exhibits a highly structured state different from a simple atomic fluid up to temperatures beyond $3 \mathrm{eV}$ $(1 \mathrm{eV}=11604 \mathrm{~K})$, having coordination numbers of silicon by oxygen atoms that increase with density in a similar way as in the solid phases [17].

The experiment was performed on the LULI2000 laser facility at the Laboratoire Pour l'Utilisation des Lasers Intenses at the Ecole Polytechnique. The experimental scheme followed the previous setup used to study the metallization of shocked and partially unloaded states of $\mathrm{SiO}_{2}$ [16]. A long-pulse laser (500 ps at FWHM of the trapezoidal shape with a rising time close to $200 \mathrm{ps}$ ) at $532 \mathrm{~nm}$ wavelength, spatially smoothed by an hybrid phase plate, was focused on a target with a quasi-flat top $400-\mu \mathrm{m}$ diameter focal spot. Both the characteristics of the spatial shape of this nanosecond pulse and its temporal shape are details in the Supplemental Material [18]. A second laser beam that delivered $15 \mathrm{~J}$ at $532 \mathrm{~nm}$ during $5 \mathrm{ps}$ with a $50-\mu \mathrm{m}$ diameter focal spot on an Ytterbium backlighter target created a spectrally flat x-ray emission from the $\mathrm{M}$ band at around $\mathrm{Si} \mathrm{K}$-edge energy, i.e. 1.85 $\mathrm{keV}$ [19]. This allowed us to probe $\mathrm{SiO}_{2}$ in a quasi-static state. The time-resolved XANES spectra were obtained by using independent KAP crystals and a double normalization between two different shots. This experimental protocol is described in detail in [20], as well as in [18], where the raw spectra are shown. Moreover, two visible rear-side diagnostics (VISAR and SOP) coupled with hydrodynamic simulations allowed us to 
extract the thermodynamic conditions shown in Fig. 1. In this experiment, the SOP system allowed us to measure the transit time of the shock in the different layers of the target, as well as the quality of the shock, as explained in $[16,21,22]$. Nevertheless, we note that the VISAR system gave us only access to the shock transit time in the all target and not to the instantaneous velocity of the shock. Indeed, the layers of diamond were not metallized below 6 Mbar [23,24] and their different interfaces induced a high level of ghost fringes. However, as thicknesses of the different layers were low, the shock stayed steady and the mean shock velocity measurements were meaningful and sufficient to give a strong constraint for hydrodynamic simulations. More details concerning the extraction of the probed thermodynamic conditions as well as of the choice of the equation of states are also presented in [18].

We used reshock compression to reach states that are more compressed and at lower temperatures than along the principal Hugoniot. This is achieved using speciallyengineered targets where we surrounded the $2 \mu \mathrm{m}$ of fused silica by $5 \mu \mathrm{m}$ of free-standing CVD diamond (see details in [16] for target fabrication). The accuracy on the thicknesses was obtained by ellipsometry $(+/-0.03 \mu \mathrm{m}$ on $\left.\mathrm{SiO}_{2}\right)$, by interferometry (+/-0.05 $\mu \mathrm{m}$ on diamond) and mechanically (1.0+/-0.5 $\mu \mathrm{m}$ of UV glue). Furthermore, a layer of graphite $(1 \mu \mathrm{m})$ was coated on the ablator to avoid direct transmission of the laser light through the rear-side diagnostics.

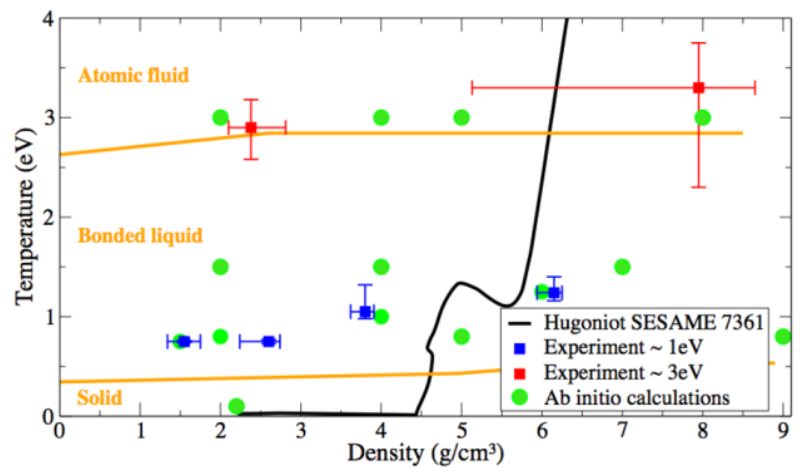

FIG. 1 (color online). Density-temperature conditions inferred from the shock timing and velocity SOP/VISAR data coupled to the hydrodynamic simulations along the isotherms $1 \mathrm{eV}$ (in blue) and $3 \mathrm{eV}$ (in red). Highly compressed $\mathrm{SiO}_{2}$ states were probed from either side of the Hugoniot. The green dots represent the conditions at which the $a b$ initio simulations were performed. The orange lines represent the boundaries of the phase diagram proposed in [13].

As shown in Fig. 1, we were able to reach denser states than along the principal Hugoniot. The error bars shown here take into account the numerical laser intensity used to reproduce the measured shock velocities (15\%), the precision of the timing probe $(50 \mathrm{ps})$, and the accuracy on the target design mentioned above. We note that for the higher densities and temperatures reached in this study, the re-shocked layer of $\mathrm{SiO}_{2}$ stays highly-compressed for a relatively short time compared to the other reached conditions, namely close to $100 \mathrm{ps,} \mathrm{which} \mathrm{explain} \mathrm{the}$ large error bars observed at these conditions. By considering partially released states, we can also investigate different densities at fairly constant temperatures conditions. From both the number of spectra and the uncertainties on their associated densitytemperature conditions, we chose to focus on the $1 \mathrm{eV}$ and $3 \mathrm{eV}$ isotherms. Figure 2 shows the spectra obtained at these conditions compared to the theoretical predictions.

We first see in Fig. 2 that the experimental XANES spectra are very sensitive to the change in density along both isotherms. As density increases, we clearly observe that the edge shifts toward higher energies (i.e blue shift), that the slope of the edge decreases, while the first maximum widens. This evolution of the overall spectrum is consistent with what is found theoretically. The theoretical calculations of the XANES spectra consist in $a b$ initio simulations using the ABINIT electronic structure code [25] and PAW pseudopotentials [26] generated using the Atompaw generator [27]. We performed the calculations on a regular grid at conditions indicated by green dots in Fig. 1, and extending to $8 \mathrm{~g} / \mathrm{cm}^{3}$. As described in more details in [16], these calculations consist in quantum molecular dynamics simulations based on density functional theory (DFT), within the generalized gradient approximation (GGA) as parameterized by PBE functional [28]. To properly describe the high densities, we generated pseudo-potentials using 4 and 6 active electrons for respectively the $\mathrm{Si}$ and $\mathrm{O}$ atoms while the corresponding PAW radius were set at $1.5 \mathrm{a}_{\mathrm{B}}$ and $1.1 \mathrm{a}_{\mathrm{B}}$. Finally, we calculated the XANES spectra using the impurity model for 10 snapshots along each trajectory, as described in [29, 30].

Following previous work done on aluminum [21], we defined i) the position of the foot of the $\mathrm{K}$ edge, $\mathrm{E}_{\mathrm{Kf}}$, by using a linear fit $\mathrm{f}(\mathrm{E})$ of the slope and its value when crossing the $\mathrm{x}$-axis $\left(\mathrm{f}\left(\mathrm{E}_{\mathrm{Kf}}\right)=0\right)$ and ii) the energy of the first maximum of the spectrum $E_{k M}$.
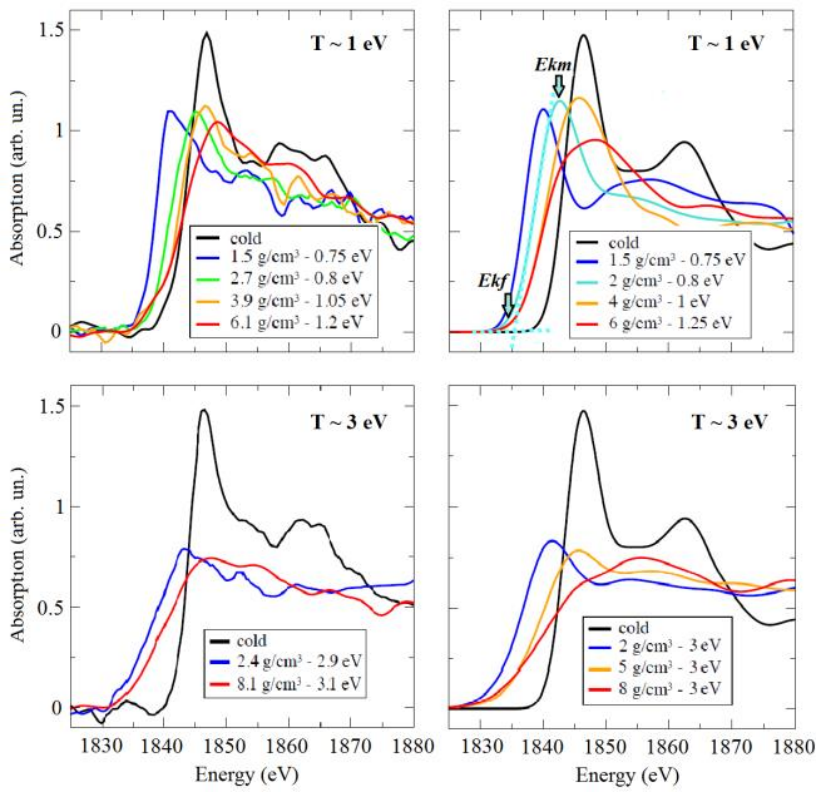

FIG. 2 (color online). Si K edge XANES spectra measured (left) and calculated (right) near $1 \mathrm{eV}$ (up) and $3 \mathrm{eV}$ (down).

Fig. 3 shows the variation of both quantities compared to normal conditions of temperature and density, 
i.e. of the cold solid $\mathrm{SiO}_{2}$, respectively $\mathrm{E}_{\mathrm{Kf}}-\mathrm{E}_{\mathrm{Kf}}^{0}$ and $\mathrm{E}_{\mathrm{KM}^{-}}$ $\mathrm{E}_{\text {Kм. }}^{0}$. The agreement between the calculations and the experiment is rather satisfactory for both isotherms. By comparing the spectrum corresponding to the cold solid $\mathrm{SiO}_{2}\left(\rho=2.20 \mathrm{~g} / \mathrm{cm}^{3}\right)$ with the others in Fig. 2, we notice a red shift when the temperature increases. This red shift was associated to the closure of the gap with temperature as extensively discussed in [16]. It explains the negative values of $E_{\mathrm{Kf}}-E_{\mathrm{Kf}}^{0}$ and $E_{\mathrm{KM}}-E_{\mathrm{KM}}^{0}$ observed in Fig. 3 . Thereafter, we are interested to compare spectra at constant temperatures. Then, as the density increases, we find that the values of the foot edge $E_{\mathrm{Kf}}-\mathrm{E}_{\mathrm{Kf}}^{0}$ do not change much : a slight displacement of a few $\mathrm{eV}$ is observed toward higher energies. In contrast, the values of $\mathrm{E}_{\mathrm{KM}}-\mathrm{E}_{\mathrm{KM}}^{0}$ are significantly shifted toward higher energies by $8 \mathrm{eV}$ and $15 \mathrm{eV}$ for respectively the $1 \mathrm{eV}$ and $3 \mathrm{eV}$ isotherms. In both calculations and experiments, the slope of the K-edge thus decreases significantly with increasing density.

To interpret this evolution of the spectra as a function of density, we first recall that the XANES is related to the unoccupied partial p-projected density of states (p-DOS). The decrease of the slope of the edge observed experimentally is related to an increase in density that causes a widening and a lowering in energy of the partial p-DOS, as well as a decrease of the pseudo-gap contrast along both isotherms. This behavior of the DOS has been previously demonstrated by ab initio calculations, which further suggest a decrease in electrical conductivity with pressure [31].
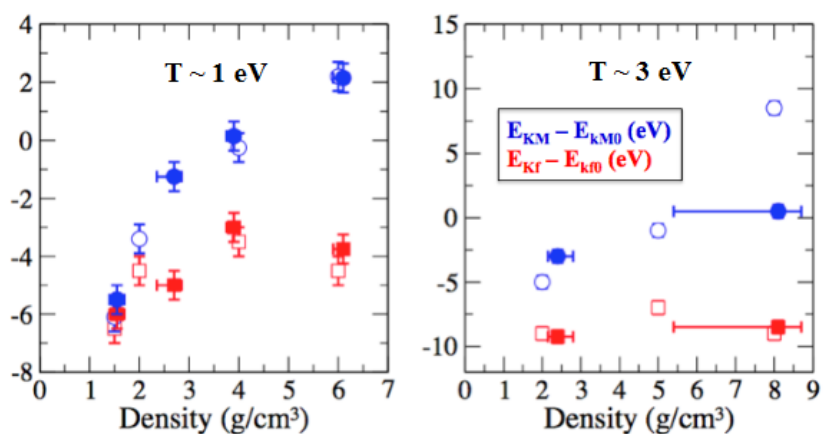

FIG. 3 (color online). Shift of the first maximum position (blue circles) and foot K-edge position (red squares) as function of density. Plain symbols are experimental data and empty symbols are calculations. Left, results along the isotherm at $1 \mathrm{eV}$; Right, results along the isotherm at $3 \mathrm{eV}$.

The strong blue shift and the modifications of the first maximum energy $E_{K M}-E_{K M}^{0}$ in the XANES spectra can be understood in terms of changes of the coordination numbers of silicon by oxygen atoms . At the density of the cold solid, the first maximum is linked to the tetrahedral structure of the Si-O bound [17]. Its shift and widening can be interpreted as a change in the ionic structure in the liquid phase that follows what was previously found for the solid phases [17]. Indeed, a clear blue shift of the Kedge is observed at ambient temperatures when the system evolves from the $\alpha$-quartz $\left(\rho=2.65 \mathrm{~g} / \mathrm{cm}^{3}\right)$, to the coesite $\left(\rho=2.92 \mathrm{~g} / \mathrm{cm}^{3}\right)$, and the stishovite phase $\left(\rho=4.29 \mathrm{~g} / \mathrm{cm}^{3}\right)$. All of these characteristics are associated to the change of the $\mathrm{Si}$-O coordination number in the solid phases in which it varies from a value of four at normal density (tetrahedral coordinence) to reach nine in the cotunnite and $\mathrm{Fe}_{2} \mathrm{P}$ phases [32]. Both the experimental measurements and the $a b$ initio predictions indicate that the XANES spectra of the liquid follow a similar evolution than the solid. This suggests that the evolution of the liquid structure at temperatures at least up to $3 \mathrm{eV}$ resembles the one of the solid.

To assess the evolution of the liquid structure with density, we first extracted the Si-O pair correlation functions, $g_{\mathrm{Si}-\mathrm{O}}(\mathrm{r})$, from the simulations. In Fig. 4-a), we show the evolution of this function along the $3 \mathrm{eV}$ isotherm and for densities ranging from 2 to $8 \mathrm{~g} / \mathrm{cm}^{3}$. The increase of the magnitude of the first maximum with density already allows us to point out to an increase in the number of oxygen atoms around the silicon sites. This is quantified by calculating the coordination number obtained by integrating the $\mathrm{g}_{\mathrm{Si}-\mathrm{O}}(\mathrm{r})$ up to $\mathrm{r}_{\mathrm{cut}}=5,0 \mathrm{a}_{\mathrm{B}}$ at the lowest densities and up to $\mathrm{r}_{\mathrm{cut}}=4.5 \mathrm{a}_{\mathrm{B}}$ at 6,7 and $8 \mathrm{~g} / \mathrm{cm}^{3}$. These cutoff values correspond to the minimum in the $g_{S i-}$ $o(r)$ following the first peak.
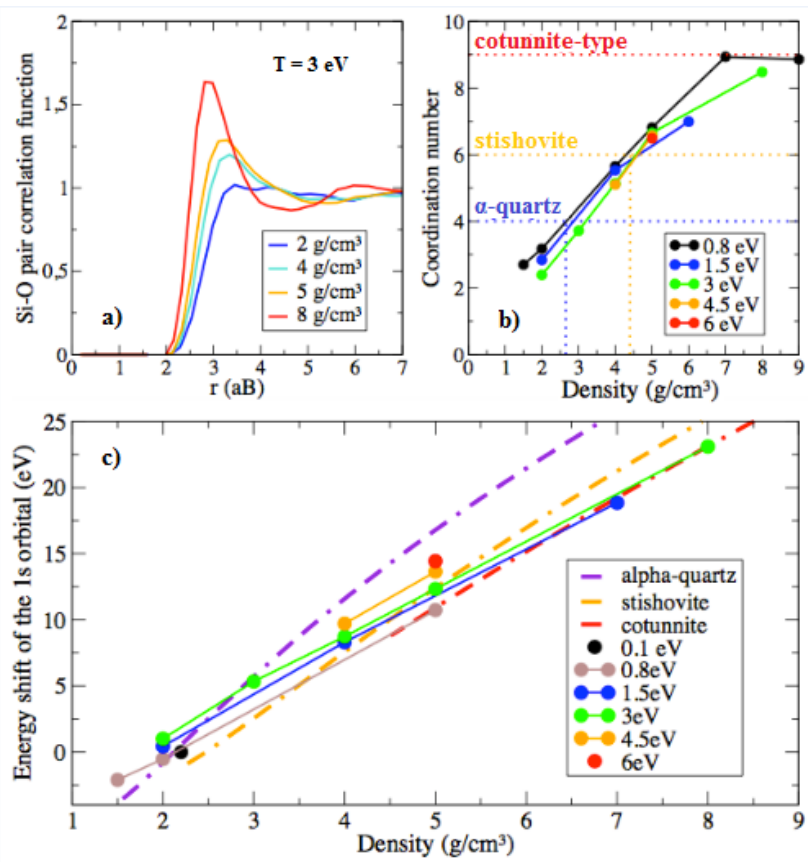

FIG. 4 (color online). (a) Variation with density of the Si-O pair correlation function along the $3 \mathrm{eV}$ isotherm given by the molecular dynamics simulations. (b) Variation of the coordination number for all the simulated conditions. Each color is associated to a temperature. (c) Energy shift of the energy of the $1 \mathrm{~s}$ orbital with the density in the solid phases (dashed lines) and in the liquid (solid lines). The temperatures are indicated in the graph.

Fig. 4-b) shows that the coordination number strongly increases with density in the liquid at all the temperatures simulated. We also notice that the temperature tends to decrease the coordination number even if its effect remains moderate. We see that the evolution of the coordination number in liquid silica follows closely what is found for the solid even at temperatures up to $3 \mathrm{eV}$.

This finding is also consistent with the agreement in the blue energy shift of the edge foot between 
experiment and calculation. Indeed, the exact position of the Si K-edge is affected by the variation of the 1s orbital energy of the silicon atom $\left(E_{1 \mathrm{~s}}\right)$ with density, as it results from the difference between this energy and the Fermi energy $\left(E_{F}\right)$. The calculation of $E_{F}$ is known and accurate, as it requires to take into account only the electrons close to the continuum [21]. On the contrary, the challenge lies in the calculation of the variation of $\mathrm{E}_{1 \mathrm{~s}}$. This is commonly refereed as the chemical shift and can be used to decipher the environment surrounding the silicon atoms. As pointed out in [28], the variation of the orbital energy with density and temperature is obtained in the simulations by going beyond the frozen-core approximation. Due to the complex nature of liquid silica, we obtain the variation of $\mathrm{E}_{1 \mathrm{~s}}$ by building an all-electron pseudopotential for the absorbing $\mathrm{Si}$ atom then used within a molecular dynamics run for each of the conditions investigated [16].

We show in Fig. 4-c) the results obtained for each density-temperature conditions investigated here. We compare these results to the evolution of $\mathrm{E}_{1 \mathrm{~s}}$ obtained for each of the solid phases occurring in this density range. We recall here that each solid phase is associated to a given coordination number of silicon by oxygen atoms. The calculations in the solid phases thus show the variation of $\mathrm{E}_{1 \mathrm{~s}}$ with density and for a fixed coordination number. For the liquid, we see that $\mathrm{E}_{1 \mathrm{~s}}$ varies from a value above the one obtained for the alpha-quartz phase at normal conditions to the value obtained for the cotunnite at around $8 \mathrm{~g} / \mathrm{cm}^{3}$ for both the $1 \mathrm{eV}$ and $3 \mathrm{eV}$ isotherms. The trend remains the same for the isotherms at higher temperatures but with values pointing toward lower coordination numbers. In this way, the good agreement found for the shift of the foot of the K-edge between the measurements and the calculations $(\leq 1 \mathrm{eV})$, which is well below the expected difference for a given density between two extreme coordination numbers $(>5 \mathrm{eV})$ is an additional indication that the coordination number of the liquid increases similarly to what is found in the solid phases. Moreover, this stands up to rather high temperatures.

In summary, we measured the XANES spectra of compressed liquid $\mathrm{SiO}_{2}$ over a large density-temperature range. With the help of $a b$ initio simulations, we interpreted the evolution of the structure of the spectra as chemical shift of the Si K edge with density as due to the evolution of the liquid toward a high coordination number of silicon by oxygen atoms close to what is found in the solid phases. These results allow us to improve the understanding of the phase diagram of the liquid $\mathrm{SiO}_{2}$ on a wide domain of density/temperature conditions in the WDM regime.

We acknowledge the expert support from the technical groups at LULI2000 facility, from the GENCI program for providing computational time, and from the french RENATECH network and its FEMTO-ST technological facility for target fabrication. This work was supported by the ANR Project PLANETLAB (ANR-12BS04-0015). The CELIA team thanks the ANR OEDYP (ANR-09-BLAN-0206-01) for equipment.
[1] I. Baraffe et al., Protostars and Planets VI (University of Arizona Press, Tucson, Arizona, 2014).

[2] G. Collins et al., Science 281, 1178 (1998).

[3] M. D. Knudson, D. L. Hanson, J. E. Bailey, C. A. Hall, J. R. Asay, and W. W. Anderson, Phys. Rev. Lett. 87, 225501 (2001).

[4] G. Huser, M. Koenig, A. Benuzzi-Mounaix, E. Henry, T. Vinci, B. Faral, M. Tomasini, B. Telaro, and D. Batani, Phys. Plasmas 12, 060701 (2005).

[5] J. Eggert, S. Brygoo, P. Loubeyre, R. S. McWilliams, P. M. Celliers, D. G. Hicks, T. R. Boehly, R. Jeanloz, and G. W. Collins, Phys. Rev. Lett. 100, 124503 (2008).

[6] I. Baraffe et al., Rep. Prog. Phys. 73, 016901 (2010).

[7] K. Umemoto, Science 311, 5763 (2006).

[8] D. K. Spaulding, R. S. McWilliams, R. Jeanloz, J. H.

Eggert, P.M. Celliers, D.G. Hicks, G.W. Collins, and

R. F. Smith, Phys. Rev. Lett. 108, 065701 (2012).

[9] J. Tsuchiya and T. Tsuchiya, Proc. Natl. Acad. Sci. U.S.A. 108, 1252 (2011).

[10] R. S. McWilliams, D. K. Spaulding, J. H. Eggert, P. M. Celliers, D. G. Hicks, R. F. Smith, G. W. Collins, and R. Jeanloz, Science 338, 1330 (2012).

[11] T. H. Di Stefano and D. E. Eastman, Solid State Commun. 9, 2259 (1971).

[12] M. D. Knudson and M. P. Desjarlais, Phys. Rev. Lett. 103, 225501 (2009).

[13] D. G. Hicks, T. R. Boehly, J. H. Eggert, J. E. Miller, P. M. Celliers, and G. W. Collins, Phys. Rev. Lett. 97, 025502 (2006).

[14] M. Millot, N. Dubrovinskaia, A. Cernok, S. Blaha, L. Dubrovinski, D. G. Braun, P. M. Celliers, G. W. Collins, J. H. Eggert, and R. Jeanloz, Science 347, 6220 (2015).

[15] Y. Laudernet, J. Clérouin, and S. Mazevet, Phys. Rev. B 70, 165108 (2004).

[16] A. Denoeud, A. Benuzzi-Mounaix, A. Ravasio, F. Dorchies, P. M. Leguay, J. Gaudin, F. Guyot, E. Brambrink, M. Koenig, S. Le Pape, and S. Mazevet, Phys. Rev. Lett. 113, 116404 (2014).

[17] D. Li, G. M. Bancroft, M. Kasrai, M. E. Fleet, R. A. Secco, X. H. Feng, K. H. Tan, and B. X. Yang, American Mineralogist 79, 622 (1994).

[18] See Supplemental Material at [] for details of the experimental setup and data analysis.

[19] M. Harmand, F. Dorchies, O. Peyrusse, D. Descamps, C. Fourment, S. Hulin, S. Petit, and J. J. Santos, Phys. Plasmas 16, 063301 (2009).

[20] A. Levy, F. Dorchies, C. Fourment, M. Harmand, S. Hulin, J. J. Santos, D. Descamps, S. Petit, and R. Bouillaud, Rev. Sci. Instrum. 81, 063107 (2010).

[21] A. Benuzzi-Mounaix, F. Dorchies, V. Recoules, F. Festa, O. Peyrusse, A. Levy, A. Ravasio, T. Hall, M. Koenig, N. Amadou, E. Brambrink, and S. Mazevet, Phys. Rev. Lett. 107, 165006 (2011).

[22] M. Harmand et al., Phys. Rev. B 92, 024108 (2015).

[23] F. Coppari et al., Nature Geoscience 6, 926-929 (2013).

[24] D. K. Bradley et al., Phys. Rev. Lett. 93, 195506 (2004).

[25] X. Gonze et al., Comput. Mater. Sci. 25, 478 (2002). 
[26] P. E. Blochl, Phys. Rev. B 41, 5414 (1990). [27] N. A. W. Holzwarth, A. R. Tackett, and G. E. Matthews, Comput. Phys. Commun. 135, 329 (2001).

[28] J. P. Perdew, K. Burke, and M. Ernzerhof, Phys. Rev. Lett. 77, 3865 (1996).

[29] S. Mazevet and G. Zerah, Phys. Rev. Lett. 101,155001(2008).

[30] V. Recoules and S. Mazevet, Phys. Rev. B 80, 064110 (2009).

[31] S. Mazevet, T. Tsuchiya, T. Taniuchi, A. BenuzziMounaix and F. Guyot, Phys. Rev. B 92, 014105 (2015). [32] T. Tsuchiya and J. Tsuchiya, Proceedings of the National Academy of Sciences 108, 1252-1255 (2011). 